Results: In all women in whom glucose decreased ( $n=138)$, insulin release was satisfactory at $1 \mathrm{~h}$. The remaining women had apparent ongoing absorption with increased incremental glucose concentration; 77 of these had insulin release at $1 \mathrm{~h}$ (no retesting required); 38 had markedly delayed glucose absorption with low initial insulin release (retesting required) and 69 showed some insulin release ongoing at $1 \mathrm{~h}$ continuing to increase at $2 \mathrm{~h}$ (immediate retesting not helpful). In a very small number $(n=8)$ neither glucose absorption nor insulin release were demonstrated.

Discussion: In 330 studied flat OGTT, we identified that reflex insulin testing was not needed in $42 \%$. Reflex insulin testing demonstrating glucose absorption saved $23 \%$ of 330 women a repeat OGTT.

\section{ANALYSIS OF QUALITY IMPROVEMENT FORMS IN DEPARTMENT OF CLINICAL CHEMISTRY IN SRI LANKAN TERTIARY CARE REFERENCE LABORATORY}

Harsha Samarasekara, Saroja Siriwardena, Sandeepani Gunawardena, Susantha Liyanage, Nadie Perera, Chandana Padmakantha

Lanka Hospital Diagnostics, Colombo, Sri Lanka

Lanka hospital diagnostics (LHD) is ISO 15189 Accredited tertiary care reference laboratory, offering general and specialised tests in clinical chemistry. In July 2014 the test menu of this laboratory was upgraded by provision of state of the art analysers and provision of high level supervision by a fulltime specialist chemical pathologist. The quality management system of LHD has also been upgraded in line with higher level of international accreditation standards such as JCIA and CAP.

Aims of this study:

1. To identify basic deficiencies (pre-analytic, analytic and postanalytic errors present in the clinical chemistry laboratory.

2. Education of all stakeholders about specific corrective and preventive measures.

3. To create a data base where regular data analysis is performed and required interventions carried out regularly.

Method: Fifty-five quality improvement forms received by clinicians and patients were analysed. Deficiencies identified during routine work were also actively reported by technical and quality team.

Results: The majority of quality improvement forms received by the whole laboratory were lodged by department of clinical chemistry (33.4\%); pre-analytical errors (30.9\%), analytical errors $(12.7 \%)$, post-analytical errors $(16.4 \%)$, no errors $(34.5 \%)$ and administrative/safety issues $(5.4 \%)$ were identified.

Common errors included following: communication gap between laboratory and clinicians, sample identification errors, report delays, sample collection errors for specialised tests, lack of clinician's contact details to inform critical values, test ordering errors, sample transport errors, transcription error, incorrect reference ranges, methodological errors, IT networking error and report formatting error.

Discussion: Each deficiency has been identified. Root cause analysis and corrective actions were done in real time. Preanalytical error rates were $<0.01 \%$, comparable with literature from developed countries. Every effort will be taken with the highest level of management commitment to prevent such errors in future. Laboratory staff, phlebotomist, nursing and all stake holders will be regularly educated.

\section{IMMERSION OF BOVINE EYEBALLS AFTER ONE HOUR IN SEAWATER DOES NOT RESULT IN ELEVATION OF POST-MORTEM VITREOUS HUMOR SODIUM AND CHLORIDE LEVELS}

\section{S. Anne, R. Tse, C. Oldmeadow, A. Cala}

Department of Forensic Medicine, Newcastle, Forensic and Analytical Science Service (FASS), NSW Health Pathology, John Hunter Hospital, Newcastle, NSW, Australia

Background: Elevations in post-mortem vitreous sodium chloride (PMVSC) levels may help differentiating seawater drowning deaths from immersion deaths not related to drowning. It is unclear whether the elevation is related to blood electrolyte changes following inhalation of seawater, or due to passive electrolyte diffusion or osmosis across the outer coats of the eye during immersion.

Aim: To investigate the changes in bovine PMVSC at different time points whilst immersed in seawater.

Methods: Forty-eight bovine eyes were obtained from an abattoir and randomised into two groups: (a) submerged in seawater ('wet group') or (b) placed in an impermeable plastic bag immersed in seawater ('dry group'). The PMVSC from six eyeballs were measured from each group (without replacement) at 30 minutes, 1 hour, 6 hours and 12 hours.

Results: There was no significant difference in mean PMVSC between the two groups at 30 minutes and 1 hour intervals. Significant difference was noted from 6 hours onwards.

Discussion: Bovine PMVSC levels remain constant when immersed in seawater for up to 1 hour. Assuming similar physical properties in humans, elevations in PMVSC in bodies immersed in seawater for less than 1 hour would not be due to passive electrolyte diffusion or osmosis across the outer coats of the eye.

\section{SUBARACHNOID HAEMORRHAGE DUE TO ANEURYSMAL RUPTURE SECONDARY TO FIBROMUSCULAR DYSPLASIA}

J.M. Dyke $^{1}$, D.M. Moss ${ }^{2,3}$, V.A. Fabian ${ }^{1,3}$

${ }^{1}$ Neuropathology, PathWest, Royal Perth Hospital, ${ }^{2}$ Forensic Pathology, PathWest, QEII, Perth, and ${ }^{3}$ School of Pathology and Laboratory Medicine, University of Western Australia, WA, Australia

Case report: A 26-year-old female was found deceased at home in bed, with subarachnoid haemorrhage identified as the cause of death on post-mortem examination. On macroscopic examination of the brain there was a small aneurysm of the anterior communicating cerebral artery with probable rupture, associated with widespread subarachnoid haemorrhage. Histology of the circle of Willis and major branches confirmed the ruptured aneurysm, secondary to a non-inflammatory 
cerebral vasculopathy in keeping with fibromuscular dysplasia (FMD).

Discussion: FMD is a rare group of non-atherosclerotic, noninflammatory, non-amyloid arteriopathies first described in 1938. ${ }^{1}$ The aetiology is likely multifactorial and is familial in up to $10 \%$ of patients. Common sites of involvement are the renal and cerebral arteries. There is a high incidence of vascular complications in FMD due to arterial dissection and aneurysm formation with rupture. Histopathologically FMD is classified according to which arterial wall layer is involved, with lesions often confined to short segments of one or two arteries. ${ }^{2}$ Extensive blocking and histological examination of cerebral arteries should be considered in cases of unexplained subarachnoid haemorrhage in the setting of non-atherosclerotic aneurysms to exclude FMD.

\section{References}

1. Sharma AM, Kline BK. The United States Registry for fibromuscular dysplasia: new findings and breaking myths. Tech Vasc Interv Radiol 2014; 17: 258-63.

2. Plouin PF, Perdu J, La Batide-Alanore A, et al. Fibromuscular dysplasia. Orphanet J Rare Dis 2007; 2: 28.

\section{SUPRACHIASMATIC CYSTIC LESION. INCIDENTAL VS PATHOLOGICAL?}

J.M. Dyke $^{1}$, G.A. Cadden ${ }^{2}$, V.A. Fabian ${ }^{1,3}$

${ }^{1}$ Neuropathology, PathWest, Royal Perth Hospital, ${ }^{2}$ Forensic Pathology, PathWest, QEII, and ${ }^{3}$ School of Pathology and Laboratory Medicine, University of Western Australia, Perth, WA, Australia

Case report: A 45-year-old man was found deceased in bed with no cause of death readily evident from the given history or gross post-mortem examination. On macroscopic examination a $12 \mathrm{~mm}$ unilocular cyst was seen in continuity with the 3rd ventricle, inferior to the anterior commissure with compression of the adjacent optic chiasm. Histology of this cyst showed it to be a prominent optic recess of the 3 rd ventricle with normal optic chiasm histology, representing an incidental autopsy finding.

Discussion: The optic recess of the 3rd ventricle was characterised anatomically by Retzius in $1900 .{ }^{1}$ It is immediately adjacent to the optic chiasm. Cystic enlargement is most commonly secondary to hydrocephalus. This can compress the optic chiasm, presenting as bitemporal hemianopia with clinical effects. Optic chiasmal symptoms due to optic recess enlargement are related to the anatomical position of the chiasm and degree of recess distension. The differential diagnosis for a cystic lesion in this location includes a congenital/developmental cyst or acquired cyst of neoplastic or infective origin. ${ }^{2}$ The anatomical relationships of the optic recess, the causes of enlargement and the differential diagnosis for a cystic lesion at this location are discussed.

\section{References}

1. Retzius C. Biologische Untersuchungen, Neue Folge IX, Tafel XII. Stockholm, Jena: Gustav Fischer, 1900.

2. Glastonbury CM, Osborn AG, Salzman KL. Masses and malformations of the third ventricle: normal anatomic relationships and differential diagnoses. RadioGraphics 2011; 31: 1889-905.

\section{CASE REPORT: CRANIAL ERYTHROID HYPERPLASIA IN A PATIENT WITH CHRONIC ALCOHOLIC LIVER DISEASE MIMICKING TRAUMATIC HEAD INJURY}

Kate Forgan-Smith, Enrico Roche, David Williams

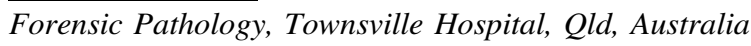

Erythroid hyperplasia (EH) represents an increase in red blood cell $(\mathrm{RBC})$ precursors in bone marrow and reflects a response to peripheral RBC loss or ineffective erythropoiesis. EH is usually seen chronic anaemias or intrinsic bone marrow disease. Less commonly, chronic hypoxaemic states or rarely erythropoietinproducing tumours can produce $\mathrm{EH}$.

We present a 42-year-old female with decompensated alcoholic cirrhosis, portal hypertension and chronic normochromic normocytic anaemia. She was found dead 5 days following an alleged assault. At autopsy for suspected homicide, she was noted to have scalp bruises and bilateral bluish discolouration over the parietal skull. Neuropathology revealed no evidence of complications of trauma to the brain. Histopathology of the parietal skull revealed EH. Death was due to complications of cirrhosis.

Bilateral EH of the parietal marrow is not recognised by the vast majority of forensic pathologists; a related condition, porotic hyperostosis is recognised by forensic anthropologists.

This case demonstrates an unusual entity, cranial EH in the setting of long-standing normochromic normocytic anaemia, related to chronic alcoholic liver disease, and also introduces the symmetrical nature of the parietal erythroid hyperplasia that may cause confusion at autopsy.

\section{VIOLET AND BLUE ALTERNATIVE LIGHT SOURCES ENHANCE VISIBILITY OF BRUISES IN CADAVERS}

Kelly Olds, Neil Langlois, Roger Byard, Calle Winskog University of Adelaide, School of Medical Sciences, Adelaide, Forensic Science SA, Adelaide, SA, Australia

Bruising is frequently documented in cases of violence for use as forensic evidence. However, bruises can be overlooked if they are not visible to the naked eye. Alternative light sources have been used in an attempt to reveal the presence of bruising that is not otherwise apparent. There is, however, a significant gap in knowledge surrounding this technique, as it has not been validated against histology to confirm that bruising is genuinely being enhanced. This is the first study evaluating the ability of alternative light sources to enhance visibility of bruises by employing histology to confirm bruising. Embalmed and nonembalmed cadavers were studied. Bodies were scanned with light sources, and enhanced regions not apparent under white light were sampled. Images were assessed using the program Fiji to measure enhancement in terms of bruise size. Photography results were compared with histology to confirm bruising. Violet and blue light sources produced the greatest enhancement, both $p<0.0001$ for embalmed and non-embalmed cadavers. Regions that were not bruises also enhanced with light sources in this study, indicating that alternative light sources are not specific. This suggests that the use of alternative 